\title{
Optimization of Sparse Learning Problem of Signals on Hybrid mm-Wave MIMO Systems using Sparse Coding based Reconstruction Learning Mechanism
}

\author{
${ }^{1}$ Sunil Kumar M., ${ }^{2}$ Narayanappa C. K., ${ }^{3}$ Nagendra Kumar M. \\ ${ }^{1}$ Research scholar, VTU Belagavi, Department of Medical Electronics Engineering, \\ Ramaiah Institute of Technology, India \\ sunilteddy@gmail.com \\ ${ }^{2}$ Associate Professor\&HoD., Department of Medical Electronics Engineering, \\ Ramaiah Institute of Technology, India \\ cknarayanappa@msrit.edu \\ ${ }^{3}$ Associate Professor,SJC Institute of Technology, India \\ mnagendrakumar@sjcit.ac.in
}

Received: January 20, 2021. Revised: July 8, 2021. Accepted: July 20, 2021. Published: July 22, 2021.

\begin{abstract}
Researchers and industry experts are looking for the availability of large bandwidth spectrum due to high market demands and expectations for high data rates. And Millimeter Wave technology possess characteristics to fulfill these requirements. However, due to high power consumption and channel estimation requirements, massive MIMO is utilized in coordination with Millimeter Wave technology. Besides, the performance of mm-WAVE MIMO system is measured by the effective estimation of Channel State Information (CSI) which is a critical and challenging process. Therefore, a Sparse Coding based Reconstruction Learning (SCL) mechanism is presented to efficiently estimate Channel State Information (CSI) for Millimeter-WAVE massive Multiple Input Multiple Output (MIMO) system. For efficiency enhancement, joint sparse learning problem is formulated and a denoised joint sparsity learning matrix is obtained using proposed SCL mechanism. Here, optimization of joint sparse learning problem is summarized by reducing inconsistent and overfitting errors. The proposed SCL mechanism performs well under high as well as low SNR conditions. Moreover, joint sparse coding algorithm is utilized for efficient sparse signal restoration. The performance of proposed SCL mechanism is efficiently measured against several stateof-art-algorithms in terms of energy efficiency, NMSE, channel capacity etc.
\end{abstract}

Keywords: Biomedical Applications, Channel State Information (CSI), Millimeter-WAVE system,massive MIMO, Sparse Coding, SNR, 5G technology

\section{INTRODUCTION}

Modern advancements in current technologies have created opportunities for the extension of technological schemes in several communication and information dependent applications which impacts various aspects of daily human life. Moreover, such technological development has impacted healthcare sector quite enormously in last two decades. However, modern medical instruments and advance healthcare devices requires high speed internet services to get benefitted from latest technology developments and for the effective implementation of diagnosis techniques such as Respiratory Diagnosis method, blood pressure measurement, Electrocardiograph (ECG) for heartbeat movement recording, Magnetic Resonance Imaging (MRI) [1]. However, these diagnosis methods produces abundant amount of valuable information which requires high storage as well as high signal transmission schemes for transfer of these valuable data to other places. And high data transmission requires high speed internet services and large scale data compression. However, current wireless communications services are not capable of transmitting these large scale of biomedical data efficiently due to bandwidth requirement expected up to some $\mathrm{GHz}$ for these scale of transmission. Therefore, several industrial experts and researchers have suggested bandwidth spectrum utilization of 5-th generation wireless communication technology as a suitable solution.

In near future, the technologies like augmented reality, autonomous driving, Internet of Services $(I O S)$ are likely to become common in daily life with lightning speed which also requires high speed internet services [2-3]. Therefore, the future 5-th generation wireless communication technology need to take an enormous leap in order to handle 
diverse requirements of several applications, domains and sectors in near future [4-7]. Moreover, it is estimated that by various experts that global rate of data traffic would enhance by 1000 times in $5 \mathrm{G}$ era which is quite enormous and concerning as well [8-9].However, it is agreed by various communication and technology experts that $5 \mathrm{G}$ is the best solution to handle ever-increasing data traffic expectations and explosive spectrum capacity demands. The utilization of $5 \mathrm{G}$ cellular network enhances scalability, energy efficiency, network throughput, packet switching and connectivity and lowers latency significantly. Many experts have suggested that a reliable $5 \mathrm{G}$ network can ensure zero delay transmission and can handle data traffic up to several billions and can ensure data rate up to some $\mathrm{GHz}$ which is quite brilliant. However, it is suggested by various experts that the future $5 \mathrm{G}$ cellular network will need at least hundreds of antenna array elements, bandwidth spectrum up to $100 \mathrm{MHz}$, ultra-densely installed source stations to support massive consumers. Moreover, numerous technical problems need to be discussed which can degrade the performance efficiency of future $5 \mathrm{G}$ cellular network such as upstream and downstream synchronization, effective topology utilization, high interference, antenna attenuation and high speed mobility environment.

Therefore, Millimeter-WAVE system is considered as one of the best possible solution to handle above mentioned issues in future $5 \mathrm{G}$ cellular network by several technology experts. The mm-WAVE system provides immense strength to 5 -th generation wireless communication technology by enhancing efficiency of high-frequency bandwidth utilization. The $5 \mathrm{G}$ wireless cellular network will require ultra-high data rates up to some gigabits-per-second in near future which requires enormous bandwidth spectrum utilization and become possible only with mm-Wave frequencies exploitation. It is stated that mm-WAVE communication system consists of 200 time bigger bandwidth spectrum in contrast to the bandwidth spectrum currently utilized by present wireless cellular networks. The mm-WAVE communication system requires only a tiny wavelength order in between 1-10 $\mathrm{mm}$ and consists a frequency band in between $30-300 \mathrm{GHz}$ in order to support high data rates [10-11]. However, large signal absorption and high antenna attenuation can degrade the performance throughput of mm-Wave communication to a large extent which can be eliminated by using several antenna array elements together in the mm-WAVE system [12].

However, the utilization of large antenna array elements can cause high power consumption in mm-WAVE system and high channel connectivity is expected. Thus, a massive Multiple Input Multiple Output (MIMO) system can employed to enhance energy efficiency of the network as well as can maintain channel connectivity between large antenna array elements. The massive MIMO system is wellequipped to recover information in low SNR conditions from Consumer Equipment $(\mathrm{CE})$ and it can reduce channel overhead to a large extent as well. Therefore, a wellcoordinated mm-WAVE MIMO system is very helpful in enhancing performance of $5 \mathrm{G}$ bandwidth spectrum. However, the performance of massive MIMO is highly dependent on efficient recovery of channel information at Source Station (SS). Moreover, proper acquisition of Channel State Information (CSI) is quite complicated process which can degrade the performance of mm-WAVE MIMO system to a large extent. CSI evaluation can be segregated into Upstream and Downstream CSI estimation. Some of the recent literatures are presented in below paragraph related to effective CSI estimation in $\mathrm{mm}-\mathrm{WAVE}$ MIMO system.

Furthermore, numerous researchers have shown interest in eliminating the issues of mm-WAVE MIMO system and provided suitable methods for the enhancement of efficiency in $5 \mathrm{G}$ cellular networks. Some of literatures are shown in below paragraph. In [13], a detailed survey is conducted over mm-WAVE communication technology, their limitations, their impact on 5G cellular network and solutions to mitigate those issues. Along with that a user association (UA) mechanism and spectrum sharing approach (SSA) is presented for performance evaluation. In [14], a Channel State Information (CSI) estimation technique is adopted for the mm-WAVE MIMO system based on hybrid beamforming antenna architecture. Along with that to decrease network overhead and enhance channel throughput an iterative beam acquisition mechanism is introduced. In [15], mm-WAVE MIMO system is adopted based on OffGrid Dirichlet Kernels to evaluate channel feedback efficiency by estimating discrete Fourier transform. The greedy low-complexity algorithms are also introduced enhance reconstruction efficiency. In [16], mm-WAVE MIMO system is introduced based on hybrid beamforming to estimate channel throughput using random spatial sampling. This technique exploits the low rank functionalities and angular data for signal reconstruction. However, some of the issues in mm-WAVE MIMO system are yet to be discussed in detail like path loss, antenna interference management, power consumption, precise CSI estimation, computational complexity reduction, effective spectral efficiency loss recovery mechanism, high channel overhead in real-time implementation which can degrade performance of mm-WAVE MIMO system and ultimately performance degradation in future $5 \mathrm{G}$ cellular network efficiency.

Therefore, a Sparse Coding based Reconstruction Learning (SCL) mechanism is adopted in this article to handle sparse learning problem encountered while Channel State Information (CSI) acquisition. In the present work, hybrid beamforming is utilized for massive Multiple Input Multiple Output (MIMO) system for the network efficiency enhancement. The proposed SCL mechanism perform supremely well under low Signal-to-Noise Ratio (SNR) and can easily exploit sparsity present in biomedical signals to ensure high quality denoised signal restoration. Furthermore, for efficiency enhancement, joint sparse learning problem is formulated and a denoised joint sparsity learning matrix is obtained using proposed SCL mechanism. The proposed SCL mechanism provide efficient solution for problem associated with sparsity learning in joint sparse coding. Finally, the proposed SCL mechanism is compared with various state-of-art-techniques 
considering several performance parameters like Normalized Mean Square Error (NMSE), SNR, channel overhead reduction, signal reconstruction efficiency and CSI estimation efficiency.

This paper is presented in following fashion which is mentioned below. Section 2, describes about the literature details presented for mm-WAVE MIMO system, their recognised limitations and how those limitations will be eliminated with the help of proposed Sparse Coding based Reconstruction Learning (SCL). Section 3 discusses about the methodology used in proposed CSI-based sparse reconstruction mechanism. Section 4 mentions about simulation results and their comparison with state-of-artscompression techniques and section 5 concludes the paper.

\section{LITERATURE SURVEY:}

It is clearly evident from recent surveys, investigations and from market demands that in near future the data rate demands will be very high and current cellular networks will not able to meet such requirements. Therefore, utilization of 5-th Generation cellular network will be adopted in near future which can ensure high data rate and meet market expectations. However, an enormous frequency spectrum will be required to meet such high data requirements. Therefore, many communication technology experts and researchers have find out that Millimeter wave communication technology is one of the most promising solutions which can help future generation communication network due to their widespread bandwidth spectrum. Furthermore, the mm-WAVE bandwidth spectrum has already been effectively utilized in next generation $5 \mathrm{G}$ radio standards [17] and several cellular network providers have decided to utilize mm-WAVE bandwidth spectrum for $5 \mathrm{G}$ cellular network. However, there are some concerning areas and issues in adopting mm-WAVE MIMO communication system which can degrade the performance of overall network. Those challenges are large energy consumption, precise CSI estimation, large computational complexity, large spectral efficiency loss, optimization problem and large channel overhead. Thus, several academy researchers have shown their interest in bringing down these issues and increase spectrum throughput. Some recent literatures are shown below in subsequent paragraph.

In [18], a sparse encoding and decoding technique is introduced for a Millimeter wave communication system to restore sparse signals from phase-less measurements. In this article, a beam alignment issue is formulated and solutions to eliminate this issue are discussed. Along with that, a sparse bipartite graph coding is also adopted. In [19], a channel estimation technique is introduced Millimeter wave communication system to utilize block sparsity and low rank structures in order to find out precise channel parameters and effectively address Doppler shift effect. An orthogonal matching pursuit algorithm is also adopted for effective signal reconstruction. In [20], a Convolutional Neural Network (CNN) based Compression Sensing (CS) technique is presented to estimate CSI feedback components for massive MIMO system. In this article, a detailed analysis over massive MIMO system, their challenges and solutions to handle those challenges are presented. In [21], deep neural network methods are adopted for mm-WAVE MIMO system based on supervised learning to handle large training data. Along with that, a multi-user hybrid beamforming structure is presented to reduce optimization problem and handle high transmission rates with lower computational complexity. In [22], a block sparse evaluation mechanism is adopted for mm-WAVE MIMO communication system to effectively estimate array steering vectors. A detailed analysis of channel estimation is presented in this article. A compression sensing technique is also introduced to channel estimation and sparsity of subcarriers. In [23], a hybrid beam former structure is introduced considering mm-WAVE MIMO system to handle secure signal transmission. A joint coding algorithm is presented for the effective handling of information leakage and to optimize channel estimation while maintain high quality of service. In [24], a highly effective precoding scheme is designed to enhance energy efficiency of mmWAVE MIMO communication system. The precoding scheme is utilized to reduce non-convex problem. A discrete connection state optimization problem is eliminated by fully-adaptive precoding scheme. In [25], a heterogeneous resource allocation mechanism is presented for mm-WAVE MIMO communication system to ensure high Quality of Service $(Q o S)$ in $5 \mathrm{G}$ cellular networks. This mechanism enhances spectrum efficiency and capacity of $5 \mathrm{G}$ Mobile Wireless Communication Network by solving optimization problem.

However, the solutions provided in above literatures are difficult to adopt in real-time implementation and few challenges remain undiscussed as well. Therefore, a Sparse Coding based Reconstruction Learning (SCL) mechanism is adopted in this article to precisely estimated Channel State Information (CSI) considering Millimeter-WAVE massive Multiple Input Multiple Output (MIMO) system and effectively handle the challenges encountered in above mentioned literatures. A comprehensive mathematical representation of proposed SCL mechanism is presented in the following section.

\section{MATHEMATICAL REPRESENTATION OF SPARSE CODING BASED RECONSTRUCTION LEARNING (SCL) MECHANISM:} In this section, a detailed mathematical modelling is presented for proposed Sparse Coding based Reconstruction Learning (SCL) Mechanism for mm-WAVE MIMO communication system to ensure high accuracy of channel estimation as well as signal restoration efficiency by eliminating sparsity learning problem associated with joint sparse coding. A detailed discussion on solutions to eliminate noise in biomedical signals is presented as well. Along with that a basic algorithm to analyze noise elimination stage, sparsity learning stage and sparsity update stage are adopted which can enhance efficiency of mmWAVE communication system. The proposed SCL mechanism reduces computational complexity to a large extent by reducing optimization problem occurs in joint sparse coding. A joint sparse coding method is utilized to exploit sparsity of biomedical signals and optimize effective 
transmission and reception of biomedical signals. The proposed SCL mechanism perform supremely well under low Signal-to-Noise Ratio (SNR) and can easily exploit sparsity present in biomedical signals to ensure high quality signal compression and signal restoration.

Here, the product of two basic steering matrices $\mathbb{T}_{W}$ and $\mathbb{T}_{Y}$ considering signals at reception and transmission side respectively gives expression for sparsity in channel matrix and defined by following equation (1) as,

$$
S_{b} \cong \mathbb{T}_{W} \chi_{b} \mathbb{T}_{Y}^{*}
$$

Where, $\mathbb{T}_{W} \triangleq\left[\mathbb{t}_{W, 0}, \mathbb{t}_{W, 1}, \ldots \ldots \ldots \mathbb{t}_{W, L_{d}-1}\right] \in \mathbb{G}^{M_{d} \times L_{d} \text { and }}$ $\mathbb{T}_{Y} \triangleq\left[\mathbb{t}_{Y, 0}, \mathbb{t}_{Y, 1}, \ldots \ldots \ldots \mathbb{t}_{Y, L_{C}-1}\right] \in \mathbb{G}^{M_{C} \times L_{c}}$ are the optimal steering matrices for receiver and transmitter respectively which need to be calculated. Here, $\chi_{b} \in \mathbb{G}^{L_{d} \times L_{c}}$ is defined as the sparse medium matrix which consists of few non-null array elements. The uncertainties between steering and medium matrixes are eliminated by the optimization of columns in steering matrix which is discussed in following equation,

$$
\begin{gathered}
\left\|\mathbb{t}_{W, l_{d}}\right\|_{2}=1, \forall l_{d} \in Q\left(L_{d}\right) \text { and }\left\|\mathbb{t}_{Y, l_{c}}\right\|_{2}=1, \forall l_{c} \\
\in Q\left(L_{c}\right)
\end{gathered}
$$

Where, number of columns in each steering matrices are defined by $L_{d} \geq M_{d}$ and $L_{c} \geq M_{c}$. Here, consider that sparse medium matrix $\chi_{b}$ produces high sparsity in biomedical signals. Thus, sparse medium matrix consists of very low uncertainties and strongly adaptable to channel conditions.

The frequency domain medium matrix at the $a \in$ $Q\left(N_{a}\right)$ subcarrier is represented as,

$$
\begin{aligned}
& S[a] \\
& =\sum_{h=0}^{M_{v^{-1}}} S_{b} e^{-u \frac{2 \pi a h}{M_{a}}} \\
& =\mathbb{Q}_{W} \delta_{W} \mathbb{E}_{W}\left(\sum_{h=0}^{M_{v^{-1}}} \eta_{h} e^{-u \frac{2 \pi a h}{M_{a}}}\right) \mathbb{Q}_{Y}{ }^{*} \delta_{Y}{ }^{*} \mathbb{E}_{Y}{ }^{*} \\
& \cong \mathbb{T}_{W}\left(\sum_{h=0}^{M_{v}-1} \chi_{h} e^{-u \frac{2 \pi a h}{M_{a}}}\right) \mathbb{T}_{Y}^{*}
\end{aligned}
$$

Where, $\quad\left(\sum_{h=0}^{M_{v}-1} \eta_{h} e^{-u \frac{2 \pi a h}{M_{a}}}\right)=\eta[a]$

and $\left(\sum_{h=0}^{M_{v}-1} \chi_{h} e^{-u \frac{2 \pi a h}{M_{a}}}\right)=\chi[a]$ defines the medium gain in frequency domain. Let all the parameters i.e. $\mathbb{T}_{W}, \mathbb{T}_{Y}, \mathbb{Q}_{W}, \mathbb{Q}_{Y}, \mathbb{E}_{W}, \mathbb{E}_{Y}, \delta_{W}, \delta_{Y}$ are frequency independent. Then,

$$
\begin{aligned}
\operatorname{vec}(S[a])=\left(\left(\overline{\mathbb{Q}_{Y} \mathbb{E}_{Y} \bar{\delta}_{Y}}\right)\right. \\
\\
\left.\otimes\left(\mathbb{Q}_{W}, \mathbb{E}_{W}, \delta_{W}\right)\right) \operatorname{vec}(\eta[a]) \\
\cong\left(\overline{\mathbb{T}}_{Y} \otimes \mathbb{T}_{W}\right) \operatorname{vec}(\chi[a]) \\
=\Omega \tilde{s}[a]
\end{aligned}
$$

Where, $\Omega \triangleq\left(\overline{\mathbb{T}_{Y}} \otimes \mathbb{T}_{W}\right) \in \mathbb{G}^{M_{d} M_{c} \times L_{d} L_{c}}$ shows the joint sparsity between biomedical signals and $\tilde{s}[a] \triangleq$ vec $(\chi[a]) \in \mathbb{G}^{L_{d} L_{c} \times 1}$ shows the vectorised steering matrices of a medium acquired with the help of joint sparse coding method and then the product of these two matrices will give $\operatorname{vec}(S[a])$.

Then, the recovered signal at Consumer Equipment (CE) for the $a-t h$ subcarrier is defined by following equation,

$$
\begin{array}{r}
\varrho[a]=P_{b b}^{*}[a] P_{r s c}^{*} S[a] E_{r s c} E_{b b}[a] i[a] \\
+P_{b b}^{*}[a] P_{r s c}^{*} \zeta[a]
\end{array}
$$

Where, $i[a] \in \mathbb{G}^{N_{i} \times 1}$ shows the vector term of transmitted signal for the $a-t h$ subcarrier.

For precise optimization of CSI and joint sparsity, the vector term of transmitted signal is further factorized as,

$$
i_{\aleph}[a]=\varpi_{\aleph} c_{\aleph}[a]
$$

Where, $\varpi_{\aleph} \in \mathbb{G}^{N_{c} \times 1}$ represent a group of frequencies with same magnitudes and $c_{\aleph}[a]$ is a complex exponential in time at receiver. Then, training pattern is determined with the help of joint sparse coding based on the supplementary signal sequenced $d_{\mathrm{m}}[a], \mathrm{m}=0,1,2 \ldots \ldots \ldots$. Then, for a predefined propagation factor $M_{\Re}$ the training pattern is defined by following equation,

$$
c_{\aleph}[a]=d_{\left|\frac{\aleph}{M_{\Re}}\right|}[a]
$$

Then, equation (7) is further factorized as,

$$
c_{\aleph}[a]=d_{0}[a], \ldots \ldots d_{0}[a], d_{1}[a], \ldots \ldots d_{1}[a], \ldots . .
$$

Then, after joining the gathered training patterns for the $a-$ th subcarrier in the $\mathbb{N}-t h$ training signals considering OFDM is determined by following equation,

$$
\varrho_{\aleph}[a]=P_{\aleph}^{*} S[a] E_{\aleph} \varpi_{\aleph} c_{\aleph}[a]+P_{\aleph}^{*} \zeta_{\aleph}[a]
$$

The product of received signal as shown in equation (10) $\varrho_{\aleph}[a]$ and $\left(c_{\aleph}[a]\right)^{-1}$ gives effective signal restoration using joint sparse coding method. Then,

$$
\begin{aligned}
\tilde{\varrho}_{\aleph}[a] \triangleq \operatorname{vec}\left(\left(c_{\aleph}[a]\right)^{-1} \varrho_{\aleph}[a]\right) \\
\left.=E_{\aleph}^{Y} \varpi_{\aleph}^{Y} \otimes P_{\aleph}^{*}\right) \operatorname{vec}(S[a]) \\
+\tilde{\zeta}_{\aleph}[a]
\end{aligned}
$$

Where $\tilde{\zeta}_{N}[a]$ is represented as $\mathrm{s} \tilde{\zeta}_{N}[a] \triangleq$ $\operatorname{vec}\left(\left(c_{\aleph}[a]\right)^{-1} P_{\aleph}^{*} \zeta_{\aleph}[a]\right)$. Then, from equation (4) and (10),

$$
\begin{aligned}
\tilde{\varrho}_{\aleph}[a] \cong\left(E_{\aleph}^{Y} \varpi_{\aleph}^{Y}\right. & \left.\otimes P_{\aleph}^{*}\right)\left(\overline{\mathbb{T}_{Y}} \otimes \mathbb{T}_{W}\right) \operatorname{vec}(\chi[a]) \\
& +\widetilde{\zeta}_{\aleph}[a]=\Delta_{\aleph} \Omega \tilde{s}[a]+\widetilde{\zeta}_{\aleph}[a]
\end{aligned}
$$

Where, $\Delta_{\aleph} \triangleq\left(E_{\aleph}^{Y} \varpi_{\aleph}^{Y} \otimes P_{\aleph}^{*}\right) \in \mathbb{G}^{N_{d} \times M_{d} M_{c}} \quad$ shows an observation matrix for $N-$ th OFDM signal evaluated with the help of pre-processors and combiners and the quantification matrix is defined by $\Delta_{\aleph} \Omega$ and evaluated for the efficient sparse signal restoration. Moreover, all the 
gathered signals are averaged out for effective sparse signal restoration without any noise.

$$
\begin{gathered}
\tilde{\varrho}_{a v g, \sigma}[a]=\sum_{\aleph=0}^{M_{\Re}-1} \tilde{\varrho}_{\sigma M_{\mathfrak{R}}+\aleph}[a] / M_{\mathfrak{R}} \text { for } \sigma \\
=0,1,2 \ldots . .
\end{gathered}
$$

Then, signals are received at the $a-t h$ subcarrier shown in the following equation and an observation matrix is determined based on the average quantification achieved above.

$$
\begin{aligned}
& {\left[\tilde{\varrho}_{\text {avg,0 }}^{Y}[a], \ldots ., \tilde{\varrho}_{\text {avg, } \mathbb{F}-1}^{Y}[a]\right]^{Y}} \\
& =\left[\Delta_{0}^{Y}, \ldots \ldots \Delta_{\mathbb{F}-1}^{Y}\right]^{Y} \Omega \tilde{s}[a] \\
& +\left[\tilde{\zeta}_{\text {avg, } 0}^{Y}[a], \ldots . ., \tilde{\zeta}_{\text {avg, } \mathbb{F}-1}^{Y}[a]\right]^{Y}
\end{aligned}
$$

Where,

$$
\begin{gathered}
\tilde{\varrho}[a] \in \mathbb{G}^{\mathbb{F} N_{d} \times 1}, \Delta \in \mathbb{G}^{\mathbb{F} N_{d} \times M_{d} M_{c}} \text { and } \tilde{\zeta}[a] \\
\in \mathbb{G}^{\mathbb{F} N_{d} \times 1}
\end{gathered}
$$

Then, a basic algorithm is adopted for sparsity learning to train proposed SCL mechanism so that efficiency of mmWAVE communication system get enhanced. The equation (15) represents the two essential parameters $\Omega$ which demonstrates joint sparsity of biomedical signals and $\tilde{s}[a]$ shows vectorised steering matrix of a medium are investigated. Then, the sparsity learning problem to optimize $\Omega$ and $\tilde{s}[a]$ are evaluated in the following equation using proposed SCL mechanism,

$$
\min _{\Omega \in \beth, \widetilde{s}[a]}\|\tilde{\varrho}[\mathrm{a}]-\Delta \Omega \tilde{s}[\mathrm{a}]\|_{2}^{2}+\mathrm{p}\|\tilde{\mathrm{s}}[\mathrm{a}]\|_{0}
$$

Where, $l_{0}$-normalization is defined by $\|\cdot\|_{0}$ which implies that regression parameter $p$ and non-zero elements present in the matrix reduces overfitting and maintains the balance of the sparsity levels in training. Then, from equation (1), the uni-norm identification elements present in the matrix are operated as uni-norm limiting parameters for joint sparsity learning and given by following equation,

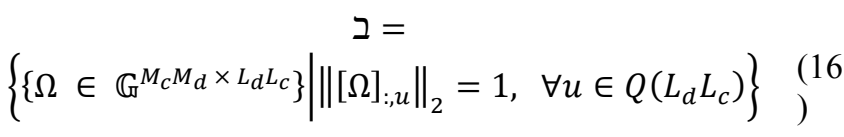

Then, from the property of vectorised steering matrix of a medium $\tilde{s}[a]$, all the elements present in the rows of vectorised steering matrix $\tilde{s}$ in equation (13) are remains either zero or non-zero and vectorised steering matrix $\tilde{s}[a]$ exhibits sparsity support between subcarriers to avoid beam polarization effect for $a \in Q\left(M_{a}\right)$. Hence, the exploitation of sparsity support between subcarriers and enhancement in number of training OFDM signals provide opportunity for $\mathrm{CE}$ to gather numerous measurement data at diversified locations. Therefore, an efficient training of SCL mechanism is carried out for various sparsity levels instead of specific regions which shows the dependency of scarification process over sparsity levels. Then, consider that $M_{i \Subset}$ measurements are gathered at diversified locations and from equation (13), every measurement $\tilde{\varrho}$ is represented as $\tilde{\varrho}^{(b)}$ where $b \in Q\left(M_{i \oplus}\right)$. Then,

$$
\begin{aligned}
{\left[\tilde{\varrho}^{(0)}, \tilde{\varrho}^{(1)} \ldots, \tilde{\varrho}^{\left(M_{i 巴}-1\right)}\right] } & \\
& =\Delta \Omega\left[\tilde{S}^{(0)}, \tilde{S}^{(1)} \ldots ., \tilde{S}^{\left(M_{i 巴}-1\right)}\right] \\
& +\left[\tilde{\zeta}^{(0)}, \tilde{\zeta}^{(1)} \ldots ., \tilde{\zeta}^{\left(\mathrm{M}_{\mathrm{ie}}-1\right)}\right]
\end{aligned}
$$

Where, $\mathbb{H}$ is defined as $\mathbb{H}=\left[\tilde{\varrho}^{(0)}, \tilde{\varrho}^{(1)} \ldots, \tilde{\varrho}^{\left(M_{i \mathrm{e}^{-1}}\right)}\right] \in$ $\mathbb{G}^{\mathbb{F N}} N_{d} \times M_{a} M_{i e}, \quad \mathbb{K}$ is defined as $\mathbb{K}=$ $\left[\tilde{S}^{(0)}, \tilde{S}^{(1)} \ldots ., \tilde{S}^{\left(M_{i \mathbb{e}}-1\right)}\right] \in \mathbb{G}^{L_{d} L_{c} \times M_{a} M_{i e}}$ and $\mathbb{R}$ is defined as $\mathbb{R}=\left[\tilde{\zeta}^{(0)}, \tilde{\zeta}^{(1)} \ldots ., \tilde{\zeta}^{\left(M_{i \mathrm{e}}-1\right)}\right] \in \mathbb{G}^{\mathbb{F N} N_{d} \times M_{a} M_{i e}}$.

Moreover, the proposed SCL mechanism performs well under low SNR conditions as well for mm-wave communication system. Here, equation (17) shows the error reduction between measurement parameters in sparse learning process using proposed SCL mechanism. Therefore, $\mathbb{H}$ is redefined by following equation,

$$
\mathbb{H}=\Delta \Omega \mathbb{K}+\mathbb{R}=\mathbb{Y}+\mathbb{R}^{\prime}
$$

Where, $\mathbb{R}^{\prime}$ is constitute of $\mathbb{R}$ and inconsistent error between $\Delta \Omega \mathbb{K}$ and $\mathbb{Y}$. Then, the inconsistent error can be reduced by adding regression term $\left(\|\mathbb{H}-\mathbb{Y}\|_{E}^{2}\right)$ in sparsity learning as,

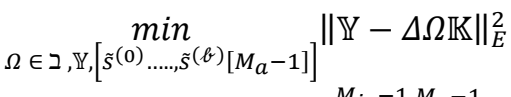

$$
\begin{aligned}
& +p_{1} \sum_{b=0}^{M_{i \mathrm{e}}-1} \sum_{a=0}^{M_{a}-1}\left\|\tilde{s}^{(b)}[a]\right\|_{0} \\
& +p_{2}\|\mathbb{H}-\mathbb{Y}\|_{E}^{2}
\end{aligned}
$$

Where, $\quad\left\{\tilde{s}^{(0)}=\tilde{s}^{(1)}=\tilde{s}^{(2)}=\cdots=\tilde{s}^{(b)}\right\}$, for all $b \in$ $Q\left(M_{i \Perp}\right)$. Then, the combination of regression term and sparsity support property can enhance sparsity between subcarriers. Thus, the joint sparse learning enhancement term in combination with sparsity support property is given by following equation,

$$
\begin{aligned}
f(\mathbb{K})=\sum_{b=0}^{M_{i \mathbb{e}^{-1}}}\left\|\tilde{S}^{(b)}\right\|_{2,1} & \sum_{b=0}^{M_{i \mathbb{e}^{-1}}} \sum_{\sigma=0}^{L_{d} L_{c}-1}\left\|\left[\tilde{S}^{(b)}\right]_{\sigma,:}\right\|_{2}
\end{aligned}
$$

Finally, the joint sparse learning problem is formulated by following equation,

$$
\min _{\Omega \in \beth, \mathbb{Y}, \mathbb{K}}\|\mathbb{Y}-\Delta \Omega \mathbb{K}\|_{E}^{2}+p_{1} f(\mathbb{K})+p_{2}\|\mathbb{H}-\mathbb{Y}\|_{E}^{2}
$$

Then, further optimization of joint sparse learning problem is achieved by updating channel steering matrix $\mathbb{K}$ while keeping other two parameters $\mathbb{Y}$ and $\Omega$ as constant. Similarly, in the next step, $\mathbb{Y}$ gets updated and other two parameters $\Omega$ and updated channel steering matrix $\mathbb{K}$ remains constant and finally, $\Omega$ gets updated while updated 
$\mathbb{K}$ and $\mathbb{Y}$ remains constant. Then, channel steering matrix $\mathbb{K}$ is updated by following equation by keeping other two parameters constant,

$$
\min _{\widetilde{\mathbb{K}}^{(b)}}\left\|\widetilde{\mathbb{Y}}^{(b)}-\Delta \Omega \widetilde{\mathbb{K}}^{(b)}\right\|_{E}^{2}+p_{1}\left\|\widetilde{\mathbb{K}}^{(b)}\right\|_{2,1}
$$

Then, the parameters of joint sparse matrix $\Omega$ gets updated for $l-t h$ coloumn while keeping $\mathbb{Y}$ and updated channel steering matrix $\mathbb{K}$ as constant and keeping other columns of the matrix constant as well. Then, joint sparsity is updated by following equation,

$$
\min _{\Omega}\|\mathbb{Y}-\Delta \Omega \mathbb{K}\|_{E}^{2}, \quad \text { s.t. }\left\|[\Omega]_{:, l}\right\|_{2}=1
$$

Finally, $\mathbb{Y}$ gets updated while keeping updated $\mathbb{K}$ and $\mathbb{Y}$ as constant to reduce inconsistent error in sparsity learning process which is defined by following equation,

$$
\min _{\mathbb{Y}}\|\mathbb{Y}-\Delta \Omega \mathbb{K}\|_{E}^{2}+p_{2}\|\mathbb{H}-\mathbb{Y}\|_{E}^{2}
$$

For the proposed SCL mechanism which requires the representation of the denoised joint sparsity which is represented in equation (24). The whole modelling process is summarized in an algorithm to understand the joint sparsity learning process using proposed SCL mechanism.

Table 1 Algorithm for Proposed SCL Mechanism

Algorithm 1: Joint Sparse Learning using Proposed SCL Mechanism

Given: $\quad$ Measurement parameter $\mathbb{H} \in \mathbb{G}^{\mathbb{F} N_{d} \times M_{a} M_{i e}}$, observation matrix $\Delta \in \mathbb{G}^{\mathbb{F} N_{d} \times M_{d} M_{c}}$, Number of training OFDM signals $M_{a}$, Number of measurements $M_{i 巴}$, regression parameters $p_{1}, p_{2}$.

Step 1: Fix joint sparse matrix $\Omega \in \mathbb{G}^{L_{c} L_{d} \times M_{c} M_{d}}$ with the help of observation matrix $\Delta$ and fix $\mathbb{H}=\mathbb{Y}$. $\begin{array}{lll}\text { Step } & 2: & \text { Evaluate }\end{array} \mathbb{K}=\left[\tilde{S}^{(0)}, \tilde{S}^{(1)} \ldots, \tilde{S}^{\left(M_{i \mathrm{e}}-1\right)}\right] \in$

Step 3: While update channel steering matrix $\mathbb{K}$

Do

Update joint sparse matrix $\Omega$ as $\Omega=$ $\Delta^{\dagger} \mathbb{Y} \mathbb{K}^{*}\left(\mathbb{K} \mathbb{K}^{*}\right)^{-1}$ and $\Omega$ gets updated column wise.

Update $\mathbb{Y}$ as $[\mathbb{Y}]=\left(1+p_{2}\right)^{-1}\left(p_{2} \mathbb{H}+\right.$ $\Delta \Omega \mathbb{K}$ ) by keeping updated $\Omega$ and $\mathbb{K}$ constant End while

Output: denoised joint sparsity learning matrix $\Omega$, denoised measurement factor $\mathbb{Y}$ and channel steering matrix $\mathbb{K}$.

Table 2. Simulation Parameters for Performance Evaluation

IV RESULT AND DISCUSSIONS:

In this section, the performance of proposed Sparse Coding based Reconstruction Learning (SCL) mechanism is evaluated to precisely estimate Channel State Information
(CSI) in comparison with various conventional CSI estimation techniques considering performance matrices like Normalized Mean Square Error, Channel delay and efficiency of the mm-Wave Communication system. The proposed SCL mechanism provide significant performance under low SNR conditions and eliminates optimization problem occurs in various conventional algorithms. A Highly effective training is achieved to determine upstream and downstream CSI components. All the experiments are performed using MATLAB $B^{T M}$ tool. For efficiency enhancement, joint sparse learning problem is formulated and a denoised joint sparsity learning matrix is obtained using proposed SCL mechanism. Along with that channel and measurement matrices are precisely examined to reduce inconsistent error and avoid overfitting between sparsity levels. The performance of proposed SCL mechanism is efficiently measured against several state-of-art-algorithms in terms of energy efficiency, NMSE, channel capacity etc. In this work, the value of Monte-Carlo simulations are kept at 100 to determine efficient simulation outcomes. A significant improvement in efficiency is observed using proposed SCL mechanism by eliminating overfitting and inconsistent error. Here, it is considered that transmitter and receiver operates with idyllic synchronization to determine CSI components. Table 2 demonstrates the parameter utilized to obtain the simulation results. Moreover, the performance of proposed SCL mechanism is determined against state-of-art-techniques such as Least Square (LS) Estimation algorithm [16], Vector Approximate Message Passing [VAMP] [26], MMV- Orthogonal Matching Pursuit (MMV-OMP) [28], Analog HBF [27] against NMSE considering various system parameters as shown in below figures.

Here, mm-Wave massive MIMO system is considered for different values of antenna elements at Transmitter and Receiver. Moreover, performance is measured using proposed SCL mechanism by estimating channel state information in time domain at receiver side. Here, analog combiner is designed with the help of Zadoff-Chu (ZC) sequence whose $Q-t h$ root value is set as $Q=11$. And fully digitalized combiner is designed by equalling number of RX RF chains to the number of antenna elements at receiver side. Here, channel steering matrix estimation performance is evaluated using proposed SCL mechanism. The performance of channel steering matrix estimation is compared with several conventional estimation techniques as a function of Normalized Mean Square Error (NMSE). The available estimation techniques works upon compression sensing techniques whereas proposed SCL mechanism evaluates performance by optimizing joint sparsity learning problem.

Here, Figure 1 shows NMSE performance considering different SNR values for various mm-Wave massive MIMO system parameters as shown in table 3. NMSE performance of proposed SCL mechanism is compared against state-ofart-algorithms like LS, MMV-OMP, VAMP and Analog $\mathrm{HBF}$. It is evident from figure 1 that LS shows much improved performance for higher values of SNR in terms of NMSE whereas VAMP and Analog HBF show decent 
results. However, the proposed SCL mechanism works

\begin{tabular}{|l|l|}
\hline Simulation Parameter & Parameter Value \\
\hline Antenna Array Characteristics & $\begin{array}{l}\text { Uniform Linear Array } \\
\text { (ULA) }\end{array}$ \\
\hline Monte-Carlo realizations & 100 \\
\hline $\begin{array}{l}\text { Signal To Noise Ratio (SNR) } \\
\text { (dB) }\end{array}$ & 15 \\
\hline Number of Frames & 40 \\
\hline Channel Delay Tap & 4 \\
\hline $\begin{array}{l}\text { Number of antenna elements at } \\
\text { Transmitter }\end{array}$ & 16 \\
\hline $\begin{array}{l}\text { Number of Propagation path per } \\
\text { channel delay tap }\end{array}$ & 6 \\
\hline
\end{tabular}

efficiently for low as well as high .

Table 3: considered simulation parameter and its values.

SNR Values. The proposed SCL mechanism outperforms all the above mentioned existing techniques in terms of NMSE considering different SNR values.

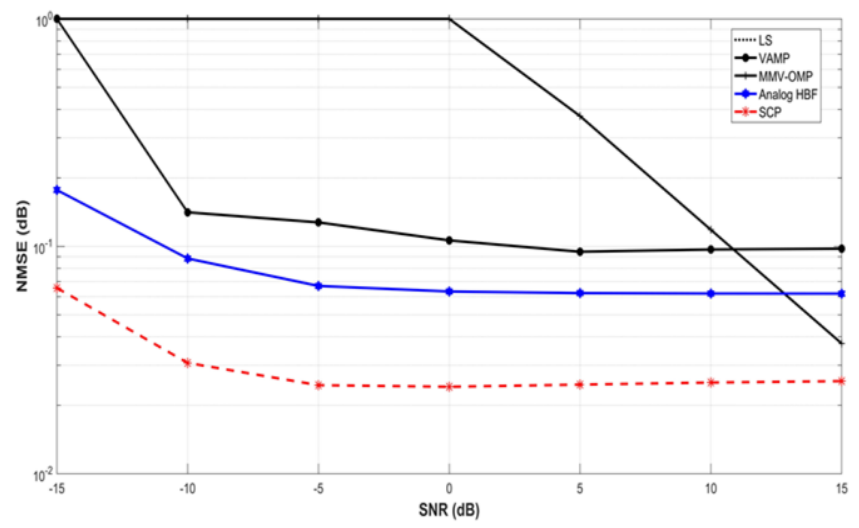

Figure 1. NMSE performance as a function of the SNR for considering antenna elements at transmitter $=8$ and receiver $=32$ and considering different parameters

Figure 2 demonstrates NMSE performance as a function of number of channel propagation paths for various $\mathrm{mm}$-Wave massive MIMO system parameters. NMSE performance of proposed SCL mechanism is compared against state-of-artalgorithms like LS, MMV-OMP, VAMP and Analog HBF at $\mathrm{SNR}=15 \mathrm{~dB}$ Here, LS and MMV-OMP consists of highest NMSE for both lower and higher values of channel path whereas VAMP gives lower NSME results than LS and MMV-OMP. And a major improvement in NMSE result is observed in Analog HBF. However, the proposed SCL mechanism gives lowest NSME results for lower as well as higher values of channel propagation path. Hence, it is evident that the proposed SCL mechanism outperforms all existing techniques in terms of NMSE for different values of channel propagation path.

Figure 3 demonstrates NMSE performance as a function of number of RF chains for various mm-Wave massive MIMO system parameters. NMSE performance of proposed SCL mechanism is compared against state-of-art-algorithms like LS, VAMP and Analog $\mathrm{HBF}$ at $\mathrm{SNR}=15 \mathrm{~dB}$ Here,
VAMP consists of highest NMSE for both lower and higher values of channel path whereas Analog HBF gives lower NSME results than VAMP. It is observed that LS performs better when number of RF chains are more. Similarly, the proposed SCL mechanism provide lower NMSE results when value of RF chains is more. Hence, from the figure it is evident that the proposed SCL mechanism outperforms all existing techniques in terms of NMSE for different values of RF chains.

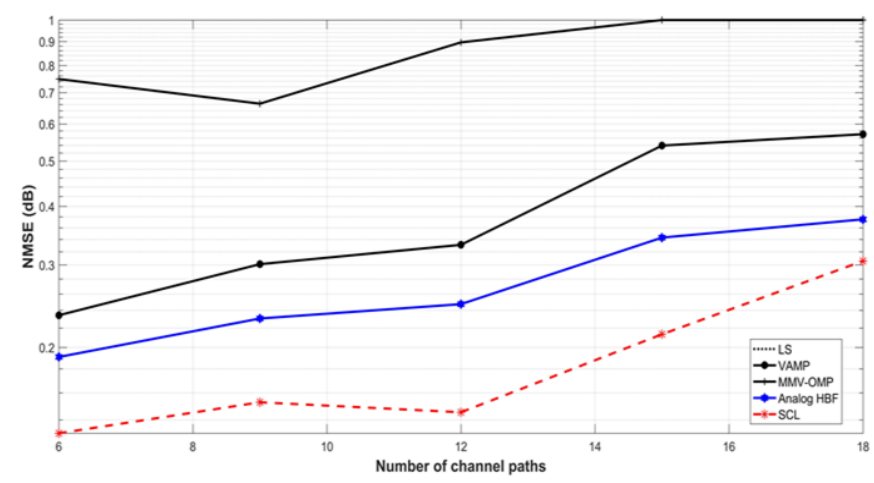

Figure 2. NMSE performance as a function of number of channel paths for considering antenna elements at transmitter $=8$ and receiver $=32$ and considering different parameters at $\mathrm{SNR}=15 \mathrm{~dB}$

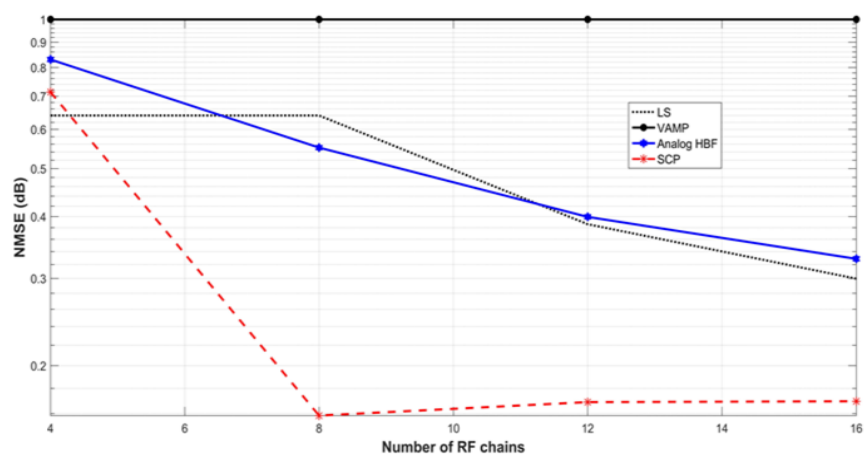

Figure 3. NMSE performance as a function of number of RF chains for considering antenna elements at transmitter $=8$ and receiver $=32$ and considering different parameters at $\mathrm{SNR}=15 \mathrm{~dB}$

Figure 4 shows NMSE performance as a function of number of channel delay taps for various $\mathrm{mm}$-Wave massive MIMO system parameters. NMSE performance of proposed SCL mechanism is compared against state-of-art-algorithms like LS, VAMP, MMV-OMP and Analog HBF at SNR $=15$ dB Here, VAMP consists of highest NMSE for both lower and higher values of channel delay taps whereas Analog HBF and LS gives lower NSME results than VAMP. It is observed that Analog HBF performs satisfactory. However, the proposed SCL mechanism provide lowest NMSE results when large number of channel delay taps are utilized. Hence, from the figure it is evident that the proposed SCL mechanism outperforms all existing techniques in terms of NMSE for different values of RF chains.

Similarly, Figure 5 shows NMSE performance as a function of number of training frames for various $\mathrm{mm}$-Wave 
massive MIMO system parameters. NMSE performance of proposed SCL mechanism is compared against state-of-artalgorithms like LS, VAMP, MMV-OMP and Analog HBF at $\mathrm{SNR}=15 \mathrm{~dB}$ Here, It is observed that LS performs better for higher number of frames. Similarly, the proposed SCL mechanism provide satisfactory results for high values of training frames.

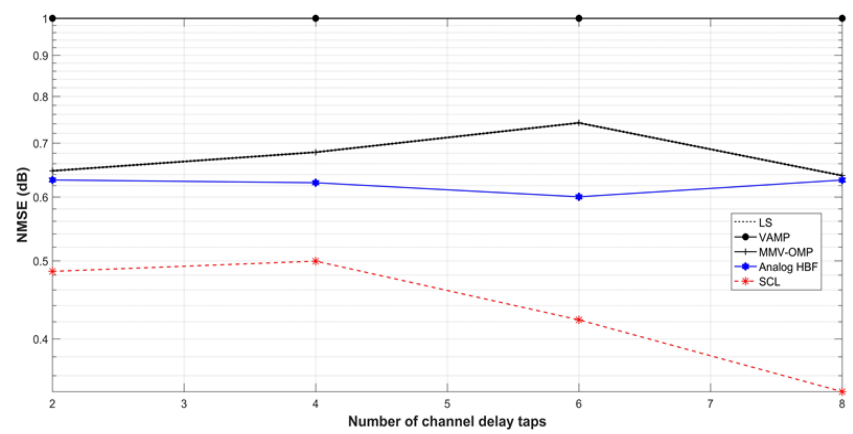

Figure 4. NMSE performance as a function of number of channel delay taps for considering antenna elements at transmitter $=8$ and receiver $=32$ and considering different parameters at $\mathrm{SNR}=15 \mathrm{~dB}$

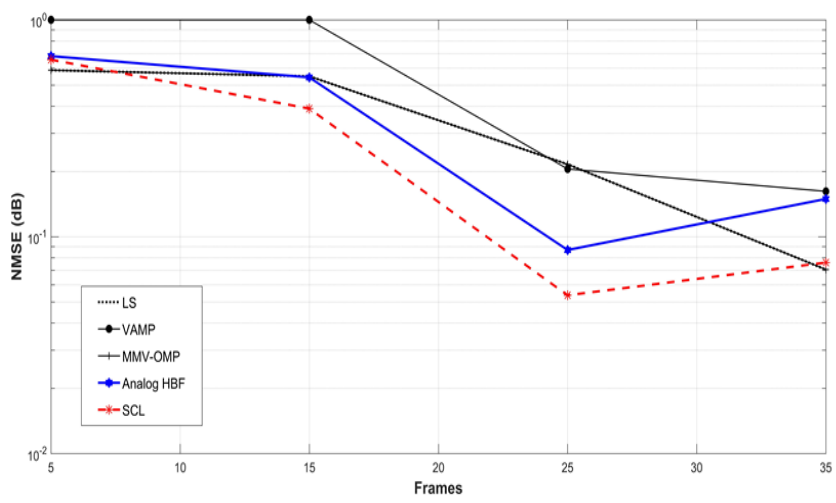

Figure 5. NMSE performance as a function of number of training frames for considering antenna elements at transmitter $=8$ and receiver $=32$ and considering different parameters at $\mathrm{SNR}=15 \mathrm{~dB}$

$$
\text { V. Conclusion: }
$$

The significance of precise Channel State Information (CSI) estimation is very essential in mm-Wave massive MIMO system for effective utilization of $5 \mathrm{G}$ bandwidth spectrum. Therefore, in this article, Sparse Coding based Reconstruction Learning (SCL) mechanism is introduced for efficient estimation of channel matrices. The proposed SCL mechanism is utilized to measure upstream and downstream CSI components at Source Station (SS) received from Consumer Equipment (CE). A comprehensive mathematical modelling for the estimation of denoised joint sparsity learning, denoised measurement and channel steering matrices are presented. The combination of joint sparse learning mechanism and $l_{0}$-normalization reduces overfitting between different sparsity levels of training. Preprocessors, combiners and the quantification matrix is utilized for effective signal restoration. The performance of channel steering matrix estimation is compared with several conventional estimation techniques considering Normalized
Mean Square Error (NMSE). The performance of proposed SCL mechanism is measured as a function of different values of Signal to Noise Ratio (SNR), number of channel propagation path, number of RF chains, number of channel delay taps and number of frames against NMSE. The proposed SCL mechanism outperforms all existing techniques in terms of NMSE considering different factors and hence efficiently estimates CSI components. As a continuation of the proposed work for the direction of the future scope it can be extended for the analysis of the work for the combinational parameters for a particular MIMO design.

\section{REFERENCES}

[1] A. Mishra, F. Thakkar, C. Modi, R. Kher, "ECG signal compression using Compressive Sensing and wavelet transform," 2012 Annual International Conference of the IEEE Engineering in Medicine and Biology Society, San Diegot, CA, 2012, pp. 3404-3407.

[2] Majed, M. B., Rahman, T. A., \& Aziz, O. A. (2018). Propagation path loss millimetr and outdoor coverage measurements review in millimetre wave bands for $5 \mathrm{G}$ cellular communications. International Journal of Electrical and Computer Engineering (IJECE), 8(4), 2254-2260.

[3] Demestichas, P., Georgakopoulos, A., Tsagkaris, K., \&Kotrotsos, S. (2015). Intelligent 5G networks: Managing 5G wireless/ mobile broadband. IEEE Vehicular Technology Magazine, 10(3), 41-50.

[4] Chedia, J., \&Belgacem, C. (2018). Performance of caching in wireless small cell networks.Journal of Telecommunication, Electronic and Computer Engineering, 10(1), 35-43.

[5] Emmanuel, A. B., Tekanyi, A., Yahaya, M., \&Gadam, M. A. (2017). Improving load balancing in various user distribution LTE advanced HetNets through a hybrid channel-gain accessaware cell selection scheme. Journal of Telecommunication, Electronic and Computer Engineering, 10(1), 1723.

[6] Sakaguchi, K., Haustein, T., Barbarossa, S., Strinati, E. C., Clemente, A., Destino, G., et al. (2017). Where, when, and how mmWaveisused in $5 \mathrm{G}$ and beyond. IEICE Transactions on Electronics, 100(10), 790-808.

[7] Alsharif, M. H., \&Nordin, R. (2017). Evolution towards fifth generation $(5 \mathrm{G})$ wireless networks: Current trends and challenges in the deployment of millimetre wave, massive MIMO, and small cells. Telecommunication Systems, 64(4), 617637.

[8] H. Peng et al., "Ultra Dense Network: Challenges, Enabling Technologies and New Trends," China Communications, vol. 13, no. 2, pp. 30-40, Feb. 2016.

[9] 5G PPP Architecture Working Group, "View on 
5G Architecture", Available: https://5gppp.eu/wp-content/uploads/2014/02/5G-PPP-5GArchitecture-WP-July-2016.pdf, Jul. 2016.

[10] Rappaport, T. S., Heath, R. W., Daniels, R. C., \&Murdock, J. N. (2014). Millimeter wave wireless communications. New York: Pearson Education.

[11] Rangan, S., Rappaport, T. S., Erkip, E., GomezCuba, F., Rappaport, T. S., \&Erkip, E. (2015). Millimeter-wave cellular wireless networks: Potentials and challenges. Proceedings of the IEEE, 102(3), 366-385.

[12] A. L. Swindlehurst, E. Ayanoglu, P. Heydari, and F. Capolino, "Millimeter-wave massive MIMO: the next wireless revolution" IEEE Commun. Mag., vol. 52, no. 9, pp. 56-62, September 2014.

[13] Attiah, M.L., Isa, A.A.M., Zakaria, Z. et al. A survey of mmWave user association mechanisms and spectrum sharing approaches: an overview, open issues and challenges, future research trends. Wireless Netw 26, 2487-2514 (2020). https://doi.org/10.1007/s11276-019-01976-X

[14] S. Chen, Q. Gao, R. Chen, H. Li, S. Sun and Z. Liu, "A CSI acquisition approach for mmWave massive MIMO," in China Communications, vol. 16, no. 9, pp. 1-14, Sept. 2019, doi: 10.23919/JCC.2019.09.001.

[15] C. K. Anjinappa, Y. Zhou, Y. Yapici, D. Baron and I. Guvenc, "Channel Estimation in mmWave Hybrid MIMO System via Off-Grid Dirichlet Kernels," 2019 IEEE Global Communications Conference (GLOBECOM), Waikoloa, HI, USA, 2019, pp. 1-6, doi: 10.1109/GLOBECOM38437.2019.9013906.

[16] E. Vlachos, G. C. Alexandropoulos and J. Thompson, "Wideband MIMO Channel Estimation for Hybrid Beamforming Millimeter Wave Systems via Random Spatial Sampling," in IEEE Journal of Selected Topics in Signal Processing, vol. 13, no. 5, pp. 1136-1150, Sept. 2019, doi: 10.1109/JSTSP.2019.2937633.

[17] 3GPP, TS 38.104 V15.0.0 Base Station (BS) radio transmission and reception (Release 15), Dec. 2017.

[18] X. Li, J. Fang, H. Duan, Z. Chen and H. Li, "Fast Beam Alignment for Millimeter Wave Communications: A Sparse Encoding and Phaseless Decoding Approach," in IEEE Transactions on Signal Processing, vol. 67, no. 17, pp. 4402-4417, 1 Sept.1, 2019, doi: 10.1109/TSP.2019.2929460.

[19] George Kiokes, "Hardware Implementation of 802.11ad MIMO-OFDM Transceiver “, WSEAS Transactions on Communications, ISSN / E-ISSN 1109-2742 / 2224-2864, Volume 18, 2019, Art. $\# 10$, pp. 71-77

[20] J. Guo, C. Wen, S. Jin and G. Y. Li, "Convolutional Neural Network-Based MultipleRate Compressive Sensing for Massive MIMO CSI Feedback: Design, Simulation, and Analysis," in IEEE Transactions on Wireless Communications, vol. 19, no. 4, pp. 2827-2840, April 2020, doi: 10.1109/TWC.2020.2968430.

[21] M. I. Youssef, A. E. Emam, M. AbdElghany, "ICI and PAPR Enhancement in MIMO-OFDM System using RNS Coding," WSEAS Transactions on Communications, ISSN / E-ISSN: 1109-2742 / 2224-2864, Volume 18, 2019, Art. \#20, pp. 145-152.

[22] M. Wang, F. Gao, N. Shlezinger, M. F. Flanagan and Y. C. Eldar, "A Block Sparsity Based Estimator for mmWave Massive MIMO Channels With Beam Squint," in IEEE Transactions on Signal Processing, vol. 68, pp. 49-64, 2020, doi: 10.1109/TSP.2019.2956677.

[23] X. Tian, Q. Liu, Z. Wang and M. Li, "Secure Hybrid Beamformers Design in mmWave MIMO Wiretap Systems," in IEEE Systems Journal, vol. 14, no. 1, pp. 548-559, March 2020, doi: 10.1109/JSYST.2019.2923819.

[24] X. Xue, Y. Wang, L. Yang, J. Shi and Z. Li, "Energy-Efficient Hybrid Precoding for Massive MIMO mmWave Systems With a Fully-AdaptiveConnected Structure," in IEEE Transactions on Communications, vol. 68, no. 6, pp. 3521-3535, June 2020, doi: 10.1109/TCOMM.2020.2979139.

[25] X. Zhang, J. Wang and H. V. Poor, "Heterogeneous Statistical-QoS Driven Resource Allocation Over mmWave Massive-MIMO Based 5G Mobile Wireless Networks in the NonAsymptotic Regime," in IEEE Journal on Selected Areas in Communications, vol. 37, no. 12, pp. 2727-2743, Dec. 2019, doi: 10.1109/JSAC.2019.2947941.

[26] P. Schniter, S. Rangan, and A. K. Fletcher, "Vector approximate message passing for the generalized linear model," in Proc. Asilomar CSSC, Pacific Grove, USA, Nov. 2016, pp. 15251529.

[27] E. Vlachos, G. C. Alexandropoulos and J. Thompson, "Wideband MIMO Channel Estimation for Hybrid Beamforming Millimeter Wave Systems via Random Spatial Sampling," in IEEE Journal of Selected Topics in Signal Processing, vol. 13, no. 5, pp. 1136-1150, Sept. 2019, doi: 10.1109/JSTSP.2019.2937633.

[28] Panagiotis Varzakas, "Estimation of Optimum Antennas Number of a Spread Spectrum MIMO System under Signal Fading", WSEAS Transactions on Computers, ISSN / E-ISSN: 1109-2750 / 2224-2872, Volume 18, 2019, Art. \#36, pp. 281-284.

\section{Creative Commons Attribution License 4.0 (Attribution 4.0 International, CC BY 4.0)}

This article is published under the terms of the Creative Commons Attribution License 4.0

https://creativecommons.org/licenses/by/4.0/deed.en_US 